\title{
Genomic diversity and phylogenetic relationships in the genus Parthenium
}

\author{
Daniel C. Ilut ${ }^{\mathrm{a}}$, Paul L. Sanchez ${ }^{\mathrm{b}}$, Denise E. Costich ${ }^{\mathrm{c}}$, Bernd Friebe ${ }^{\mathrm{d}}$, Terry A. \\ Coffelt $^{\mathrm{b}}$, John M. Dyer ${ }^{\mathrm{b}}$, Matthew A. Jenks ${ }^{\mathrm{e}}$, Michael A. Gore ${ }^{\mathrm{a}, *}$ \\ ${ }^{a}$ Plant Breeding and Genetics Section, School of Integrative Plant Science, Cornell \\ University, Ithaca, NY, 14853, USA \\ ${ }^{b}$ USDA-ARS, U.S. Arid-Land Agricultural Research Center, 21881 North Cardon Lane, \\ Maricopa, AZ, 85138, USA \\ ${ }^{c}$ International Maize and Wheat Improvement Center (CIMMYT), Texcoco, Mexico \\ ${ }^{d}$ Wheat Genetics Resource Center, Department of Plant Pathology, Kansas State \\ University, Manhattan, KS, 66506, USA \\ ${ }^{e}$ Division of Plant and Soil Sciences, West Virginia University, Morgantown, WV 26506, \\ $U S A$
}

\begin{abstract}
Guayule (Parthenium argentatum A. Gray) is a perennial woody shrub native to the North American Chihuahuan Desert that holds promise as a sustainable source of natural rubber and hypoallergenic latex. The improvement of guayule for commercial-scale production could be accelerated through genomics-assisted breeding, but such a strategy is severely limited by the paucity of available genomic tools and well-characterized genetic resources. To that end, we used genotyping-by-sequencing (GBS) to simultaneously identify and genotype tens of thousands of single-nucleotide polymorphism (SNP) markers across 62 plant samples from seven wild and cultivated guayule, three Parthenium interspecific hybrid, four mariola (Parthenium incanum Kunth), and one wild quinine (Parthenium integrifolium L.) accession(s) that have been characterized for ploidy level and nuclear genome size in this and a prior study. Phylogenetic analysis using the SNP data identified at least two distinct sources of guayule breeding material in the cultivated accessions, previously unknown multi-species
\end{abstract}

\footnotetext{
${ }^{*}$ Corresponding author

Email addresses: dci1@cornell.edu (Daniel C. Ilut), paul.sanchez@ars.usda.gov (Paul L. Sanchez), d.costich@cgiar.org (Denise E. Costich), friebe@ksu.edu (Bernd Friebe), terry.coffelt.arc@gmail.com (Terry A. Coffelt), john.dyer@ars.usda.gov (John M. Dyer), majenks@mail.wvu.edu (Matthew A. Jenks), mag87@cornell.edu (Michael A. Gore)
}

Preprint submitted to Industrial Crops and Products

July 9, 2015 
hybrids within two Parthenium hybrid cultivars, and guayule/mariola hybrids within one guayule and one mariola wild collected accession. Similar to previously reported results for guayule and mariola, we observed aneuploidy and multiple ploidy levels among individual plants (mixed ploidy) within three Parthenium interspecific hybrid accessions newly characterized in this study. Nuclear genome size characterization of wild quinine, a first for this species, found an estimated haploid nuclear genome size $(5757 \mathrm{Mb})$ for the tetraploid $(2 n=4 x=72)$ accession that was more than 1.5-fold larger than that of tetraploid $(2 n=4 x=72)$ guayule or mariola. Together, these results further underscore the need for a comprehensive characterization of available guayule germplasm and sister taxa with both SNP markers and flow cytometry, illustrate the novel utility of GBS for the genus Parthenium, and lay the foundation for genomics-assisted breeding in guayule.

Keywords: Guayule, Mariola, Parthenium, Genotyping-by-sequencing, Polyploidy, Apomixis, Nuclear genome size

\section{Introduction}

Natural rubber, one of the essential raw materials of modern society, is a major international trade commodity with worldwide imports of 8.5 million metric tons valued at over 24 billion USD in 2013 (DESA/UNSD, United Nations Comtrade database; http://comtrade.un.org). Nearly all of it is derived from the rubber tree [Hevea brasiliensis (Willd. ex A. Juss.) Müll. Arg.] with more than two thirds being produced in Thailand and Indonesia (FAOSTAT database; http://faostat3.fao.org). The geographical concentration of natural rubber production and the vulnerability of monoculture rubber tree plantations to South American leaf blight caused by the fungal pathogen Microcyclus ulei

\footnotetext{
Abbreviations

GBS: genotyping-by-sequencing, GRIN: Germplasm Resources Information Network, SNP: single-nucleotide polymorphism, NCBI: National Center for Biotechnology Information, NPGS: USDA-ARS National Plant Germplasm System, NALPGRU: USDA-ARS National Arid Land Plant Genetics Resources Unit
} 
(Rivano, 1997) make the nearly exclusive reliance on $H$. brasiliensis plantations for the world's natural rubber needs a precarious situation. In combination with the projected shortage of rubber by the next decade (Mann, 2009), this has led to a renewed interest in the development of alternative sources of natural rubber.

The woody perennial shrub guayule (Parthenium argentatum A. Gray), a native plant of the desert regions of northern Mexico and southwestern United States, has been intermittently improved and cultivated as a source of natural rubber over the past century (reviewed in Hammond and Polhamus, 1965; Thompson and Ray, 1989). However, development of high rubber yielding cultivars has met with only partial success, in large part due to a combination of limited genetic diversity in the source of germplasm (Thompson and Ray, 1989), extensive polyploidy variation within and among the available guayule accessions (Gore et al., 2011), and a complex mode of reproduction that is characterized by sporophytic self-incompatibility in diploid plants and facultative apomixis (diplospory type) in polyploid plants (reviewed in Ray et al., 2005; Thompson and Ray, 1989). Recently, Gore et al. (2011) successfully adapted automated flow cytometry methods to efficiently characterize ploidy level variation in guayule breeding programs. However, in order to enable modern genomicsassisted breeding approaches for the accelerated improvement of this nascent crop, a thorough characterization of both polyploidy variation and genetic diversity within the guayule germplasm pool is essential.

In addition to rubber content, improvements in other agronomically important traits such as disease resistance, cold tolerance, biomass, and vigor could significantly contribute to the suitability of guayule as a commercial crop. Although guayule is the only species in the genus Parthenium that produces significant quantities of natural rubber, interspecific hybrids with other Parthenium species are a potential source of such agronomic improvements (reviewed in Ray et al., 2010; Thompson and Ray, 1989), and several Parthenium species such as mariola (Parthenium incanum Kunth) and wild quinine (Parthenium integrifolium L.) have been shown to form natural and/or artificial interspecific 
hybrids with guayule (Powers and Rollins, 1945; Rollins, 1946; Hashemi et al., 1989). Mariola, a perennial shrub native to Mexico and southwestern United States (Rollins, 1950), is a facultative apomict with a natural ploidy series and considered the closest related taxon to guayule (Powers and Rollins, 1945). It has been suggested as a source of vigor and disease resistance (Thompson and Ray, 1989), and its habitat range (Figure B.5) suggests a broader climatic tolerance than guayule. Wild quinine is a perennial herbaceous plant native to central and eastern United States (Mears, 1975; Rollins, 1950) and a potential source of cold resistance and non-arid adaptation (Thompson and Ray, 1989). However, with the exception of a recent ploidy and nuclear genome size evaluation of mariola accessions (Sanchez et al., 2014), minimal genomic information is available for these two Parthenium species, and the availability of this information is expected to greatly facilitate interspecific breeding programs.

Building upon previously published data for 10 guayule, mariola, and Parthenium hybrid accessions (Gore et al., 2011; Sanchez et al., 2014), we used flow cytometry to expand ploidy and nuclear genome size characterization to four interspecific hybrids between guayule and other Parthenium species. Additionally, we estimated the nuclear genome size and ploidy level of a wild quinine accession, a first for this Parthenium species. We also used genotyping-bysequencing (GBS; Elshire et al., 2011) for simultaneously identifying and scoring tens of thousands of single-nucleotide polymorphism (SNP) markers on 62 plant samples of the 15 Parthenium accessions that captured a range of ploidy levels to i) characterize genomic diversity among and within these Parthenium species and interspecific hybrids, ii) identify cryptic hybrids, and iii) estimate the heterozygosity level of Parthenium accessions.

\section{Materials and methods}

\subsection{Plant material and growth conditions}

Achenes (hereafter seeds) of one wild collected accession (R1110) and three interspecific hybrids of guayule and other Parthenium species (CAL-1, CAL-2, 
CAL-3) were obtained from the USDA-ARS National Plant Germplasm System (NPGS; www.ars-grin.gov/npgs) through the National Arid Land Plant Genetics Resources Unit (NALPGRU) in Parlier, CA. In addition, commercial seed of a wild quinine accession was obtained from Prairie Nursery, Inc., Westfield, WI, because this taxon was not available from NPGS. The pedigrees for all accessions in this study are summarized in Figure 1, and more extensive information is available in Table A.2 and references therein. Plants were grown as described in Sanchez et al. (2014). All experimental procedures that do not differ significantly from Sanchez et al. (2014) are described in AppendixC for reference.

\subsection{Collection locales and habitat range}

Collection locales for the Powers 1942 historic accessions 4254 and 4265 were identified from literature: "area southwest of Mapimí, Durango, Mexico" (Powers, 1942; Bergner, 1946) and "from 5 plants growing in an isolated area along the road from La Mancha to Pasaje" (Powers, 1942; Johnson, 1950) respectively. Locales relevant to collection sites or accession development were geolocated using the Google Maps Javascript API v1.3 (https://developers. google.com/maps/documentation/javascript), and areas of germplasm collection were geocoded using locale descriptions or recorded GPS coordinates. Maps of herbarium specimen collections for mariola, guayule, and wild quinine were obtained from a genus treatment by Rollins (1950), and approximate habitat ranges were derived by expanding outward from each collection point. A map of the northernmost guayle habitat line by Bullard (1946) was obtained from its reproduction in Hammond and Polhamus (1965). The collection maps and guayule habitat line were overlaid on a geographic map of North America using the Google Maps Javascript API v1.3.

\subsection{Sample collection and analysis of ploidy level with flow cytometry}

Leaf tissue samples were collected and prepared for ploidy analysis for each of the five accessions (CAL-1, CAL-2, CAL-5, R1110, wild quinine) as part of 
an experiment that also included 17 additional guayule, mariola, and interspecific hybrid accession(s) (Sanchez et al., 2014) following procedures described therein. A total of 12 or 15 plants were evaluated for each of the five Parthenium accessions, and samples were run in random order by assigning a plant from each accession with equal probability to one of 15 blocks (runs) on the flow cytometer. Only 12 plants were evaluated for the wild quinine accession due to its relatively lower germination and seedling survival rate. The entire experiment was replicated twice, for a total of two measurements per plant, and the ploidy level was calculated as described in Sanchez et al. (2014).

\subsection{Cytological preparation and image analysis}

Following Gore et al. (2011), we selected representative plants within a ploidy level of the five accessions and conducted mitotic chromosome counts in root tip cells as described in Sanchez et al. (2014). Counts of metaphase chromosomes from images were independently confirmed by two people (authors Paul L. Sanchez and Bernd Friebe).

\subsection{Sample preparation and analysis of nuclear genome size with flow cytometry}

Leaf tissue samples were collected and prepared for genome size analysis from 3, 4, or 6 plants each of the five Parthenium accessions, for a total of 22 plants. This analysis was performed as part of an experiment that had also included 12 additional guayule, mariola, interspecific hybrid, sunflower, and lettuce accessions (Sanchez et al., 2014) following procedures described therein.

\subsection{Sample collection, preparation, and DNA sequencing}

Leaf tissue samples consisting of multiple young leaves for each plant were collected from 62 single plants and the tissue was lyophilized for long-term storage. For each plant sample, $17 \mathrm{mg}$ to $22 \mathrm{mg}$ of lyophilized leaf tissue was ground separately with a Talboys High Throughput Homogenizer (Troemner LLC, Thorofare, NJ, USA) and total genomic DNA was isolated from the 
powdered lyophilized leaf tissue using the DNeasy Plant 96 Kit (Qiagen Incorporated, Valencia, CA, USA). The isolated genomic DNA was prepared according to sequencing center instructions (http://www.biotech.cornell.edu/brc/ genomic-diversity-facility/gbs-sample-submission). Briefly, to ensure that the isolated total genomic DNA samples were neither contaminated nor degraded, $100 \mathrm{ng}$ of each sample was run on a $1 \%$ agarose gel with $500 \mathrm{ng}$ of two $\lambda$ HindIII size/mass standards. Trial digestions with the EcoRI restriction enzyme were performed on $10 \%$ of the samples, and sample concentration was quantified using the Quant-iT PicoGreen dsDNA Assay Kit (Life Technologies, Carlsbad, CA, USA). The DNA samples for the 62 plants were arrayed on a 96 well plate into 83 sequencing samples as follows: i) 42 plant samples were placed in one well each using $750 \mathrm{ng}$ of DNA per well, producing 42 sequencing samples; ii) 17 plant samples were placed in two distinct wells each as technical replicates using $750 \mathrm{ng}$ of DNA per well, producing 34 sequencing samples; iii) 2 plant samples were placed in two distinct wells each as technical replicates using $750 \mathrm{ng}$ of DNA for the first well and $625 \mathrm{ng}$ of DNA for the second well, producing 4 sequencing samples; iv) 1 plant sample was placed in three distinct wells as technical replicates using $750 \mathrm{ng}$ of DNA per well, producing 3 sequencing samples. One well was left blank as a negative control, and the remaining 12 wells were populated with Parthenium samples not included in this study.

The DNA samples were sequenced at the Cornell Genomic Diversity Facility (Cornell University, Ithaca, NY, USA) using the GBS procedure of Elshire et al. (2011) with minor modifications. Briefly, for each sequencing sample total genomic DNA was digested with the EcoT22I restriction enzyme and Illumina Read 1 sequencing primers plus a barcode adapter were then ligated to the fragment ends. After adapter ligation, GBS libraries were generated by PCR amplification, and the resulting libraries were sequenced on a single Illumina HiSeq 2000 flow cell (Illumina Incorporated, San Diego, CA, USA). 


\subsection{GBS data processing}

All raw data processing and downstream analysis of GBS sequences, including de-novo reference assembly, SNP calling, genotyping, heterozygosity level estimation, and phylogenetic inference, was performed in a UNIX environment (Linux Mint 17; www.linuxmint.com). Custom scripts for Bash (version 4.3.11) and Perl (version 5.18.2) were used unless otherwise specified. FASTQ formatted (Cock et al., 2009) raw sequence data consisting of 101 bp long nucleotide sequences (reads) and corresponding quality values was processed by assigning each read to a sequencing sample and evaluating reads based on nucleotide sequence quality, content, and frequency. Only reads that were assigned to a specific sample and passed all of the evaluation steps (hereafter 'filtered reads') were used in subsequent analysis. Sequence evaluation criteria were optimized for de-novo reference assembly and SNP genotyping.

Raw sequence data was first demultiplexed following standard procedures (AppendixC.8). Due to variation in the length of the sample-specific sequence tags, the resulting reads ranged in length from 86 to $92 \mathrm{bp}$ and were further trimmed to a maximum length of 86 bp by removing nucleotides from the end of the read as necessary. For each trimmed read, the corresponding quality values were trimmed as well. The sequencing quality of each read assigned to a sequencing sample was evaluated, and only reads that met all of the following criteria were kept for further analysis: i) the quality value for the last base of the read must not be the special Illumina code '2', corresponding to unreliable nucleotide assignments at the end of the read; ii) the read must not contain any nucleotides encoded as ' $\mathrm{N}$ ', corresponding to unknown nucleotides; iii) the read must not contain homopolymers (i.e., sequence of identical bases) longer than 9 $\mathrm{bp}$; iv) the average quality value for the read must be $\geq 30$, corresponding to an average probability of erroneous base call per nucleotide of $\leq 0.001$; and v) the read must not contain low complexity regions longer than $25 \%$ of the read length based on the empirical distribution. Low complexity regions were identified with the DustMasker (Morgulis et al., 2006) software component of the National Center for Biotechnology Information (NCBI) C++ Toolkit (version 12.0.0; ftp: 
//ftp.ncbi.nih.gov/toolbox/ncbi_tools++/CURRENT/) using a window length of $86 \mathrm{bp}$, a threshold level of 20 , and a linker value of 1 .

The reads that met all of the sequencing quality criteria above were further evaluated for sequence content and frequency of sampling in order to identify and eliminate potential contamination, sequencing error, and reads derived from multiple genomic loci (e.g. transposable elements). A FASTA format (Lipman and Pearson, 1985) file containing the subset of distinct nucleotide sequences was generated, and for each distinct sequence the number of times it was observed (read depth) was calculated by counting the number of reads having that particular sequence. In order to reduce the possibility of sequencing error (AppendixC.7) a minimum read depth of two, corresponding to two independent reads confirming the sequence, was required for each distinct sequence, and any distinct sequences that did not satisfy this criterion were eliminated from further analysis. Distinct sequences with a read depth of two or higher were screened against the nucleotide sequences in the NCBI UniVec dataset (build 8; http://www.ncbi.nlm.nih.gov/tools/vecscreen/univec), which among other vector sequences contains entries for the specific PCR vectors used in the GBS library preparation protocol (UniVec identifiers 'gnl|uv|NGB00362.1:161' and 'gnl|uv|NGB00360.1:1-58'). They were further screened against the NCBI artificial vector sequence dataset (taxon id 29278; http://www.ncbi.nlm. nih.gov/Taxonomy/Browser/wwwtax.cgi?mode=Info\&id=29278), the guayule chloroplast sequence (NCBI gi 281190713; Kumar et al., 2009), and the "Representative Genomes" subset of the NCBI Microbial Genomes database (build of May 28, 2014; ftp://ftp.ncbi.nlm.nih.gov/blast/db/Representative_Genomes. *tar.gz). The screening step was done using the blastn component of BLAST+ (version 2.2.28; Camacho et al., 2009) with the 'blastn-short' task preset, and a successful match required $90 \%$ identity over the matched region and an E-value of 0.01 or less. All distinct sequences matching any of these target data sets were removed from further analysis. Finally, we identified in the remaining data set the top $0.01 \%$ most frequently observed distinct sequences and removed them as potential multi-locus genomic sequences. 


\subsection{De-novo genomic reference assembly}

In order to facilitate SNP identification, a de-novo guayule pseudo-reference was created using the two plant samples from the diploid guayule accession W6 429. All the filtered reads from these two samples were combined and, following Ilut et al. (2014), distinct sequences were aligned to each other using the global alignment algorithm implemented by SlideSort (version 2; Shimizu and Tsuda, 2011). Allowing for a maximum pairwise edit distance of 20 and considering both forward and reverse matches, alignments were generated using the default scoring matrix and edit distances calculated by SlideSort were further adjusted as follows: i) potential insertion-deletion polymorphisms (indels) were encoded as single events by assigning a value of 1 to the cost of an indel and removing from the calculated edit distance any contribution from the length of the indel sequence; ii) indel penalties due to global alignment were removed by modifying the edit distance such that indels at the edges of the sequences were ignored. Next, for a range of maximum pairwise edit distances $(d)$ from 1 to 20, distinct sequences with adjusted pairwise edit distances less than $d$ were grouped together into putative locus clusters using transitive clustering. For each value of $d, 90 \%$ or more of the putative locus clusters had four or fewer distinct sequences. Given that at most four alleles can be expected at a locus when aligning reads from two diploid samples, putative locus clusters were further limited to those containing 4 or fewer distinct sequences, and for each value of $d$ from 1 to 20 the distribution of pairwise distance within each of these putative locus clusters was evaluated. Regardless of the maximum threshold $d$, $80 \%$ or more of the reads within a putative locus cluster differed by 3 or fewer mismatches, and therefore the set of putative locus clusters corresponding to $d=3$ was selected for the de-novo pseudo-reference assembly. For each putative locus cluster, the distinct sequence with the highest read depth was selected as a representative nucleotide sequence. 


\subsection{SNP identification and genotype assignment}

We created a reference index from the de-novo guayule pseudo-reference sequences using the 'panati-build' component of the PANATI package (http: //panati.sourceforge.net; Ilut et al., 2012; Spindel et al., 2013) with a word size parameter of 12 . Next, for each plant sample, we aligned all reads from that sample that passed quality control against the reference index and generated genotype calls using the 'panati' and 'combine-samples' functions of the PANATI package. The software parameters used applied the following filters to the input sequences: i) trim edge bases with quality of 30 or less, ii) remove sequences with a probability of error $\geq 1 \%$ after trimming, iii) and remove sequences of $<75 \mathrm{bp}$ length after trimming. The alignment parameters required that at least $80 \%$ of the query sequence was aligned and there were at most $5 \%$ mismatched bases between query and target sequence. The SNP identification parameters required a maximum posterior error probability for a SNP call of $10^{-12}$ and a maximum posterior error probability for a genotype call of $1 \%$. We retained only biallelic SNPs, excluding from the genotype matrix all indels and SNPs with more than 2 alleles. The top $5 \%$ most polymorphic reference loci, representing loci with more than 10 SNPs per locus (Figure B.4), were also removed from the genotyping process.

\subsection{Estimation of heterozygosity levels}

In order to improve confidence in homozygous genotype calls, the genotype matrix was modified such that only genotype calls that had a read depth of at least 10 were considered to be genotyped in a particular sample. A SNP was considered heterozygous in a sample if two alleles were detected, and the level of heterozygosity for a given sample was calculated as the number of heterozygous genotype calls divided by the total number of aligned nucleotides. For polyploid samples, reads from multiple copies of the genome were considered together and the calculated heterozygosity was not modified to account for the ploidy level or relative frequency of allelic loci (gene dosage). 


\subsection{Phylogenetic network analysis}

For the phylogenetic analysis, the genotype matrix was modified such that only SNPs which had at least 4 reads from a given plant sample aligned to them were considered to be genotyped in that sample, and only SNPs that were genotyped in at least $70 \%$ of the plant samples were retained. The genotype calls were re-coded as pairs of binomial characters, using 00 for genotypes homozygous for the reference allele, 11 for genotypes homozygous for the alternative allele, and 01 for heterozygous genotypes. The re-coded genotype matrix was then converted into a concatenated character matrix, saved as a NEXUS format (Maddison et al., 1997) file, and imported into SplitsTree (version 4.1.1; Huson and Bryant, 2006). A phylogenetic network was computed by the NeighbourNet (Bryant and Moulton, 2004) distance method. For each pair of samples, the distance metric was computed as the number of characters in the re-coded genotype matrix at which the two samples differed divided by the total number of characters genotyped in both samples. Variance was estimated via the ordinary least squares method, bootstrap support was calculated using 1000 replicates, and network splits with bootstrap support below $90 \%$ were removed.

\section{Results and discussion}

\subsection{Sampling rationale, species selection, and genotyping}

The guayule germplasm accessions available in the NPGS collection span over 70 years of cultivar development and collection from wild populations. We report here for the first time a genetic characterization of plant samples from this collection with SNP markers. Multiple guayule and interspecific guayule hybrid accessions were selected from a temporal transect of cultivar development and wild collection programs between 1942 and 2008 as a representative sample of the genetic diversity available in the germplasm collection (Figure 1). Additionally, we selected for characterization recent wild collections of mariola, the Parthenium species most closely related to guayule (Rollins, 1950; Kumar et al., 2009) and a common guayule interspecific hybridization partner in the 
wild (Rollins, 1950). Contemporary nursery material of wild quinine, a Parthenium species distantly related to guayule (Rollins, 1950; Kumar et al., 2009), was selected as the outgroup for phylogenetic analysis. A subset of these accessions has been previously analyzed for ploidy, chromosome number, and nuclear genome size (Gore et al., 2011; Sanchez et al., 2014) and this study expands those analyses to a further five accessions, providing ploidy, chromosome number, and nuclear genome size information for the accessions that were genetically characterized with SNP markers (Table 1). From the 62 plant samples analyzed via GBS, we obtained 48 million reads that passed quality control. Of these reads, 2.4 million were derived from the two samples of diploid guayule (accession W6 429), and they were assembled into a de-novo pseudo-reference of 76991 putative loci, each 86 bp in length. This pseudo-reference was used to align all 48 million reads and identify 30051 variable loci with 10 or fewer SNPs per locus, for a total of 93558 SNP markers.

From this total set of SNP markers and associated genotype calls, we generated three distinct subsets suited for different measurements and analysis methods. The first subset, consisting of all genotype calls that had a read depth of 10 or higher, was used for heterozygosity level estimates since the read depth at each genotype call is critical for the reliability of homozygous genotype inference. The number of SNP markers with the required depth was variable among samples (Table A.3), but all 93558 SNP markers had the required depth in at least one sample. The second subset, consisting of 19043 SNP markers that were genotyped in at least $70 \%$ of the samples with a read depth per sample of 4 or higher, was used for phylogenetic analysis because genotyping each locus across many samples is crucial to the reliability of inferred relationships among samples. Finally, the third subset of 3330 high confidence SNP markers, defined as markers genotyped in at least $70 \%$ of the samples with a read depth of 10 or higher was not used for analyses in this study but rather generated as a potential resource for guayule breeders to construct a custom SNP genotyping array and provided as an online supplemental file. 


\subsection{Genomic characterization of guayule germplasm}

A network of phylogenetic relationships, when combined with site collection and breeding records, serves as a tool to provide evolutionary insights into genetic similarities as well as reveal inconsistencies and potential misclassifications in the identity of germplasm bank accessions. Phylogenetic network analysis of the 62 plant samples in this study identified four major groups, with the majority of samples representing guayule cultivars and wild collections clustered in group I (Figure 2). This group can be generally divided into four distinct clades, marked in Figure 2 with the letters A, B, C, and D at the root node of each respective clade.

Clade A contains the two plant samples of diploid guayule (W6 429), all the samples from accession 11591, the tetraploid samples of N566, and all the samples of the wild collected accession R1110. It should be noted that accessions 11591 and N566 were both derived from the same historic accession (4265-I; Thompson and Ray, 1989), which in turn represented one of three predominant types identified by Johnson (1950) based on morphological and biochemical characteristics of historic accession 4265 (Figure 1). Accession 4265 was derived from a bulk seed collection of five individual plants from eastern Durango, Mexico in 1942 (Figure 3). Therefore 4265-I, the accession that gave rise to both 11591 and N566 as well as another 13 of the 26 core USDA guayule accessions (Thompson and Ray, 1989), likely captured a very limited number of alleles. The W6 429 accession is likewise derived from a selection obtained during the same collection expedition (1942) and from roughly the same geographical area (Figure 3; Bergner, 1946; Thompson and Ray, 1989). Given the historical origins of these three accessions their genetic similarity is not surprising, and it is probably representative of the original germplasm collected in 1942 from Durango, Mexico.

The fourth accession in clade A, R1110, was collected in the wild in 1979 from Mesa, AZ, an area that is not part of the native range for guayule (Figure B.5; Rollins, 1950). However, Arizona has been an area of active guayule cultivation and germplasm development by both private and public entities since 
the early 20th century (Hammond and Polhamus, 1965). Beginning in 1914, the University of Arizona operated several experimental agricultural stations in Mesa and surrounding areas (University of Arizona Agricultural Experiment Station records. Special Collections, University of Arizona Libraries). Although all activities from these stations were transferred in 1983 to the Maricopa Agricultural Center in Maricopa, AZ, the experimental stations were still active when accession R1110 was collected in 1979. Together with the genetic similarity between this accession and historical guayule breeding lines, this suggests that plants in accession R1110 are derived from historic breeding lines rather than native stands of wild guayule. The plants in this accession were originally identified as a hybrid of guayule and P. tomentosum var. stramonium (Green) Rollins based on morphological characteristics, but the phylogenetic placement of R1110 in clade A rather than the clade containing artificial hybrids between guayule and P. tomentosum var. stramonium (clade D, below) does not support this particular hybrid classification.

As previously reported (Sanchez et al., 2014), ploidy level variation exists within the 11591 and N566 accessions, with 11591 containing triploid and pentaploid samples and N566 containing tetraploid and pentaploid samples. Accession 4265, which served as the source of these two accessions, was originally characterized as a triploid (Johnson, 1950), although chromosome counts higher than 54 (the triploid chromosome number for guayule) have been reported for this accession (Gerstel, 1948; Johnson, 1950). In facultatively apomictic plants such as guayule, generation of progeny with increased chromosome numbers can occur when the unreduced megaspore mother cell is fertilized (reviewed in Thompson and Ray, 1989, Table 4.1).Within accession 11591, the low frequency occurring pentaploid sample is genetically very similar to the triploid samples, suggesting a change in ploidy from triploid to pentaploid as a result of fertilization between plants from this or a closely related accession rather than hybridization with a different accession or species. Somewhat surprisingly, the tetraploid samples of accession N566 are genetically distinct from other members of clade A, including 11591, yet the accession was derived from the same 
historic accession as 11591. Whether the tetraploid samples of N566 are the result of selection of uncharacterized tetraploids from the source germplasm, or the change from triploid to tetraploid occurred later within the accession, the phylogenetic placement suggests that the pollen donor responsible for the ploidy increase was genetically distinct from the plants from which 11591 was derived. Whereas 14 of 15 samples evaluated for ploidy from accession 11591 were triploid, the predominant ploidy level for N566 (12 of 15 samples) appears to be pentaploid (Table A.4; Sanchez et al., 2014) and all the pentaploid samples of N566 in this study form a single clade distinct from clade A (Figure 2, Clade B), sharing at least 1578 SNP alleles that do not exist in any of the tetraploid samples from this accession. This suggests that pentaploid samples of N566 were the result of hybridization between tetraploid N566 plants (which belong to the A clade) and plants from an accession outside the A clade, a hybridization that also resulted in a change of ploidy. This is consistent with heterozygosity level estimates (Table 1), which show that pentaploid plants of N566 have slightly higher allelic diversity than the pentaploid plant of 11591.

Clade $\mathrm{C}$ consists of samples from the breeding line AZ-5 developed by the University of Arizona and the USDA (Ray et al., 1999), the wild collected accession R1109 from Coahuila, Mexico, and one individual from accession CFS-21 collected in the wild near Fort Stockton, Texas. Within this group, individual plant samples from accession AZ-5 are genetically more similar to plant samples from R1109 than to other AZ-5 plants. Likewise, plants from R1109 are genetically more similar to plants from AZ-5. The clade is homogeneous in terms of cytogenetic and genomic features, with all samples being tetraploid and having very similar nuclear genome sizes and heterozygosity level estimates (Table 1). As a whole, clade $\mathrm{C}$ is genetically distinct from historic guayule accessions 11591, N566, and W6 429 (clade A), containing 759 shared SNP alleles that are not found in any of the samples from clade A. Combined, these features suggest that both accessions were derived from a recent common ancestor, and that the two accessions are genetically indistinguishable.

In order to explain how accessions from such disparate sources could be so 
closely related genetically, it is important to understand the historical context of their collection and maintenance. Accession R1109 was collected in the wild in September 1976 by R. Rollins near the locality of Parras in the southern part of Coahuila, Mexico (Figure 3; Table A.2) and deposited into the National Plant Germplasm System in 1989 by Dr. D. D. Rubis along with 134 other lines from his collection. For AZ-5, the oldest traceable source of germplasm consists of selections made by Rubis from a mix of USDA lines and unknown sources, with open-pollinated seed of these plants subsequently planted at Marana, AZ, in 1982 and 1983 (Figure 3; Dierig, 1987). After Dr. Rubis' retirement, openpollinated seeds from 6 selected plants in this field were collected in 1986 and planted at Maricopa, AZ, in 1988. Further rounds of single-plant selections of open-pollinated seed and progeny evaluation based on rubber and resin content resulted in the release of six guayule breeding lines (including AZ-5) in 1997 (Ray et al., 1999). Given that R1109 was collected in 1976, it is likely that this or another related accession was already maintained by Rubis prior to the 1982 and 1983 transplants that eventually resulted in the AZ-5 accession. It should also be noted that accession R1109 had been classified as a hybrid of guayule and mariola based on morphological characteristics. However, the phylogenetic analysis in this study, which included samples of mariola, does not support this hybrid classification.

Clade C also contains one of the four plants from accession CFS-21 that were collected in the wild in Pecos County, TX, on October 29, 2008 (Figure 3; Table A.2). Although this location is more than 350 miles $(563 \mathrm{~km})$ away from the collection location for R1109 (the other wild collected accession in this clade), previous sampling of guayule specimens (Figure B.5; Rollins, 1950) indicated the presence of guayule plants throughout the intervening region. Therefore, we cannot exclude the possibility that this sample and those from R1109 are part of a large interbreeding population. However, another possible source of this CFS-21 germplasm consists of a former guayule research station originally operated by Texas A\&M University in partnership with the Firestone Tire and Rubber Company (and subsequently Bridgestone Corporation after its aquisi- 
tion of Firestone Tire and Rubber Company in 1983). The site is located near Fort Stockton, TX, less than 100 miles $(161 \mathrm{~km})$ away from the collection locale of CFS-21. Although guayule research at the site was discontinued in the late 1990s, Bridgestone Corporation continues to operate the site and records for samples collected at that location in 2005 describe the field as follows: "The Firestone Natural Rubber and Latex Company (a division of the Firestone Tire and Rubber Company) established 180 acres of guayule in 1978-1981 by transplants originating from several seed sources. Approximately 15 acres contained 23 USDA and Anderson [accession 593] lines, while 163 acres were devoted to stands derived from bulk seed collected in native populations of north-central Mexico, and from bulk collections at the Los Angeles State and County Arboretum, Arcadia, CA. About 2 acres were devoted to research plots. Firestone closed the site in 1981, and the Texas Agricultural Experiment Station leased the area in 1983 and conducted research there until 1997." (NPGS collection records for CFS18-2005 [PARL 805]). The vast majority of plantings appear to have been derived from north-central Mexico collections, likely from the germplasm collected by R.C. Rollins in 1976 (which included accession R1109) and Naqvi \& Hanson in 1977 (reviewed in Thompson and Ray, 1989). Thus, it is plausible that plants with an allelic composition similar to those of R1109 were introduced in Texas at this location and subsequently spread into the wild, either displacing or admixing with native guayule plants. Finally, given that we only found one sample from this accession with a genotype similar to AZ-5 and R1109, it is also possible that a sample from one of those two accession was erroneously labeled as CFS-21. Following up on these results, we plan to genotype further samples from accession CFS-21 and other wild collected guayule from the area in a future experiment. However, regardless of the status of this particular sample, the historical presence of north-central Mexican guayule germplasm at the Firestone plantation indicates that such guayule genotypes are expected to be present in the area.

The last major clade in Group I, clade D, consists of triploid and tetraploid samples from accessions CAL-1, CAL-2, and CAL-5. Ploidy level variation was 
detected among the CAL-5 samples of this clade, and aneuploidy was identified in all three accessions (Table 1). These accession were generated via interspecific crosses between diploid guayule germplasm and $P$. tomentosum var tomentosum DC, $P$. fruticosum Less., and $P$. tomentosum var stramonium respectively, and progeny were selected for resistance to Verticillium wilt (Estilai, 1985; Tysdal et al., 1983). The crosses were produced at the Los Angeles State and County Arboretum using a common diploid guayule maternal parent (Waln et al., 1983) and therefore many alleles are expected to be shared among them, resulting in the complex phylogenetic relationships identified in this clade. Compared to the triploid samples, the three tetraploid samples of these interspecific hybrids are more closely related to the guayule clades, suggesting an increase in ploidy resulting from a maternal interspecific hybrid being fertilized by haploid pollen from the diploid guayule parent. It should be noted that accession CAL-5, which is the source of all three tetraploid samples, was developed from openpollinated seed of early generation, segregating progeny plants that were grown alongside the diploid parents for 3 to 4 years prior to seed selection, providing ample opportunity for further crossing with the diploid parent.

\subsection{Genomic diversity among wild mariola samples}

The mariola germplasm sampled for this study (Figure 2, Group III) was all collected at the same time and from the same geographical region (Figure 3). With the exception of one plant (CFS01-2005 pentaploid in Group II), the phylogenetic analysis places all of these samples into a single clade, with samples from the same location (same germplasm identifier) and of the same ploidy level forming monophyletic groups. All the samples analyzed are likely pure mariola accessions, and the phylogenetic and collection data suggest that they are part of a large interbreeding population of wild mariola growing in southwest Texas. Although aneuploidy and variation in ploidy levels were both detected among these samples (Table 1), close phylogenetic relationships of samples with different ploidy levels (Figure 2, Group III) and high similarity in heterozygosity levels across ploidy levels (Table 1) suggest that ploidy changes 
resulted from the fertilization of unreduced megaspore mother cells by pollen of mariola plants from the same population. The nuclear genome size of mariola appears to be slightly larger than that of guayule at equivalent ploidy levels (Table 1). Furthermore, we detected a small but significant (t-test, $P<10^{-6}$ ) difference between the average chromosome size of mariola (mean of $97.98 \mathrm{Mb} /$ chromosome) and guayule (mean of 88.19 Mb / chromosome) when considering samples with exact chromosome counts (Table A.3; Figure B.6).

\subsection{Cryptic Parthenium interspecific hybrids}

Unlike traditional bifurcating phylogenetic trees, a phylogenetic network presents all conflicting phylogenetic relationships concurrently, highlighting putative hybrid samples by their well-supported placement as sister to two or more other taxa. Several samples (Figure 2, Group II) were thus identified as containing such hybrid genotypes. Four samples, previously classified as wildcollected guayule (CFS-21) or wild-collected mariola (CFS01-2005), appear to be hybrids between guayule and mariola. Given the sympatric distribution of guayule and mariola in the wild (Figure B.5; Rollins, 1950) it is not surprising that wild-collected samples of guayule and mariola contain morphologically cryptic hybrids.

In addition, four samples from interspecific guayule hybrid lines (CAL-1, CAL-2) appear to be the result of further hybridization with an unknown lineage, resulting in an increased ploidy level from triploid to pentaploid (Table 1, Figure 2). This suggests that hybridization and corresponding increase in ploidy level occurred via fertilization of an unreduced megaspore mother cell of the interspecific CAL-1 and CAL-2 hybrids by pollen from a triploid or tetraploid plant of unknown species. Estimates of heterozygosity levels (Table 1) show that these four pentaploid samples have the highest levels of any samples evaluated in this study, further supporting the hypothesis of a subsequent hybridization event. 


\subsection{Nuclear genome size and allelic divergence of wild quinine}

The wild quinine accession was obtained from a commercial nursery and represents a member of the Parthenium genus that is not limited to arid lands, but is found throughout the Eastern half of the United States (Figure B.5; Rollins, 1950). The long private branch of this clade in the phylogenetic analysis (Figure 2) indicates that it is genetically quite divergent from both guayule and mariola. The haploid nuclear genome size for tetraploid P. integrifolium is 5757 $\mathrm{Mb}$, much larger than that of guayule $(3071 \mathrm{Mb})$ or mariola (3433 Mb) plants with the same number of chromosomes (Table A.3, Figure B.6 A). Although its adaptation to colder climates presents it as a tempting candidate for improving the cold resistance of guayule germplasm, the large divergence and difference in nuclear genome size suggest successful hybridization between this species and guayule would be difficult.

\subsection{Genomic diversity within the germplasm collection}

Our results show that ploidy variation in several accessions is closely related to genetic differentiation between ploidy levels, suggesting further separation of germplasm from these accessions in order to maintain genetically consistent cultivars. In contrast with the genetic heterogeneity observed in some of the accessions, other distinct accessions show high genetic similarity, indicating that further genomic characterization of the extant wild and cultivated Parthenium germplasm pool is necessary to construct a genetically representative diversity panel for genome-wide association studies in guayule. Such a panel would significantly reduce redundancy in effort and maximize potential payoffs for future genetic studies and crop improvement efforts in this species. Specifically, SNP genotyping of other accessions derived from 4265-I should help elucidate the historic gene flow among these closely related accessions and identify the most genetically diverse subset. Similarly, the SNP genotyping of other accessions from Rubis' collection would further refine the pedigree of AZ-5 and other cultivars derived from the 1982 single-plant selections and help identify sources of novel alleles from wild collections subsequent to the Emergency Rubber Project 
of the 1940s. Genotyping of other Parthenium species with SNP markers, including the non-guayule parents of the CAL-1, CAL-2, and CAL-5, would help identify the unknown species involved in generating the pentaploid samples from these accessions as well as quantify the genetic contribution of guayule and other species in these interspecific hybrids. Finally, in order to assess the potential for novel germplasm present in wild populations of guayule from the Big Bend area of Texas, SNP genotyping of wild collections of guayule from this area as well as accessions derived from the Rollins and Naqvi \& Hanson collections should help elucidate these relationships and assess the degree of admixture between introduced guayule plants from north-central Mexico and native Texas plants.

\section{Conclusions}

This study represents the first use of GBS for the simultaneous discovery and scoring of SNP markers in Parthenium germplasm, and the first nuclear genome size estimation for wild quinine, a Parthenium species with a haploid nuclear genome size almost twice as large as guayule. We have characterized the genomic diversity of several accessions of guayule germplasm and other Parthenium species, identified the 1976 guayule collections by R. C. Rollins from the southern part of Coahuila, Mexico as the likely source of the AZ-5 cultivar, and have shown that they provided novel alleles compared to McCallum's 1942 collections from central Durango, Mexico from which most classic cultivars of guayule are derived. We identified several plants of unknown hybrid parentage that were sampled from CAL-1 and CAL-2 cultivars, as well as sampled mariola/guayule hybrid plants from among the wild collections of these species. Additionally, we demonstrated the utility of GBS for the phylogenetic classification of Parthenium accessions even in the absence of a reference genome sequence. Finally, we have shown the need for further SNP genotyping of guayule germplasm in order to optimize genetic studies and breeding efforts in guayule. 


\section{Data availability}

All sequence data has been deposited under BioProject PRJNA289254 in the NCBI Sequence Read Archive (SRA; Wheeler et al., 2008). For each plant sample, the corresponding SRA accession number is indicated in Table A.3. Nucleotide sequences for all the de-novo assembled reference loci, unfiltered genotype calls, and locus sequences and SNPs for the high confidence SNP dataset are provided as an online supplemental data set.

\section{Acknowledgements}

We thank the National Arid Land Plant Genetics Resources Unit at Parlier, CA, USA for providing seeds. Also, we wish to thank Greg Leake, Stephanie Johnson, and Kristen Cox for assistance in maintaining plants in the greenhouse and collecting leaf tissue for flow cytometry experiments. Preparation of leaf tissue samples for nuclear genome size analysis was carried out by Patrick Redmond and Michael Boerman at the Institute for Genomic Diversity at Cornell University, and instrument coordination by Lavanya Sayam at the Biomedical Sciences Flow Cytometry Core Laboratory at Cornell University. Preparation of total genomic DNA samples for GBS was carried out by Catherine B. Kandianis and Elodie E. Gazave in the Gore laboratory at Cornell University. This work was funded and supported by the USDA-ARS and USDA-NIFA/DOE Biomass Research and Development Initiative (BRDI) Grant No. 2012-10006. Mention of trade names or commercial products in this publication is solely for the purpose of providing specific information and does not imply recommendation or endorsement by the United States Department of Agriculture. The USDA is an equal opportunity provider and employer.

\section{References}

Arumuganathan, K., Earle, E. D., 1991. Estimation of nuclear DNA content of plants by flow cytometry. Plant Molecular Biology Reporter 9 (3), 229-241. 36 
Bergner, D. A., 1946. Polyploidy and aneuploidy in guayule. United States Department of Agriculture Technical Bulletin (918). 5, 14

Bryant, D., Moulton, V., 2004. Neighbor-Net: an agglomerative method for the construction of phylogenetic networks. Molecular Biology and Evolution $21(2), 255-265.12$

Bullard, W. E., 1946. Climate and guayule culture. United States Department of Agriculture, unpublished report. 5, 33

Camacho, C., Coulouris, G., Avagyan, V., Ma, N., Papadopoulos, J., Bealer, K., Madden, T. L., 2009. BLAST+: architecture and applications. BMC Bioinformatics 10, 421. 9

Cock, P. J. A., Fields, C. J., Goto, N., Heuer, M. L., Rice, P. M., 2009. The Sanger FASTQ file format for sequences with quality scores, and the Solexa/Illumina FASTQ variants. Nucleic Acids Research 38 (6), 1767-1771. 8

Costich, D. E., Friebe, B., Sheehan, M. J., Casler, M. D., Buckler, E. S., 2010. Genome-size variation in switchgrass (Panicum virgatum): flow cytometry and cytology reveal rampant aneuploidy. The Plant Genome Journal 3 (3), 130-141. 35

Dierig, D. A., 1987. Contribution of yield components to rubber production in guayule. Doctoral dissertation, University of Arizona. 17

Doležel, J., Greilhuber, J., Suda, J., 2007. Estimation of nuclear DNA content in plants using flow cytometry. Nature Protocols 2 (9), 2233-2244. 35

Elshire, R. J., Glaubitz, J. C., Sun, Q., Poland, J. A., Kawamoto, K., Buckler, E. S., Mitchell, S. E., 2011. A robust, simple genotyping-by-sequencing (GBS) approach for high diversity species. PLOS ONE 6 (5), e19379. 4, 7

Estilai, A., 1985. Registration of Cal-5 guayule germplasm. Crop Science 25 (2), 369. 19 
Gerstel, D. U., 1948. Mid-year report of the genetics section, rubber plant investigations. United States Department of Agriculture, unpublished report. 15

Gore, M. A., Coyle, G., Friebe, B., Coffelt, T. A., Salvucci, M. E., 2011. Complex ploidy level variation in guayule breeding programs. Crop Science 51 (1), 210216. $3,4,6,13,34,36$

Hammond, B. L., Polhamus, L. G., 1965. Research on guayule (Parthenium argentatum), 1942-1959. United States Department of Agriculture. 3, 5, 15, 33

Hashemi, A., Estilai, A., Naqvi, H. H., 1989. Intersectional hybrids between induced tetraploid guayule (Parthenium argentatum) and natural tetraploid P. intergrifolium (Asteraceae). Botanical Gazette 150 (1), pp. 78-83. 4

Huson, D. H., Bryant, D., 2006. Application of phylogenetic networks in evolutionary studies. Molecular Biology and Evolution 23 (2), 254-267. 12

Ilut, D. C., Coate, J. E., Luciano, A. K., Owens, T. G., May, G. D., Farmer, A., Doyle, J. J., 2012. A comparative transcriptomic study of an allotetraploid and its diploid progenitors illustrates the unique advantages and challenges of RNA-seq in plant species. American Journal of Botany 99 (2), 383-396. 11

Ilut, D. C., Nydam, M. L., Hare, M. P., 2014. Defining loci in restriction-based reduced representation genomic data from nonmodel species: sources of bias and diagnostics for optimal clustering. BioMed Research International 2014, e675158. 10

Johnson, B. L., 1950. High rubber yielding selections from a natural population of guayule. Agronomy Journal 42 (7), 345-350. 5, 14, 15

Kumar, S., Hahn, F. M., McMahan, C. M., Cornish, K., Whalen, M. C., 2009. Comparative analysis of the complete sequence of the plastid genome of Parthenium argentatum and identification of DNA barcodes to differentiate Parthenium species and lines. BMC Plant Biology 9 (1), 131. 9, 12, 13 
Lipman, D. J., Pearson, W. R., 1985. Rapid and sensitive protein similarity searches. Science 227 (4693), 1435-1441. 9

Loureiro, J., Rodriguez, E., Doležel, J., Santos, C., 2007. Two new nuclear isolation buffers for plant DNA flow cytometry: a test with 37 species. Annals of Botany 100 (4), 875-888. 34, 36

Maddison, D. R., Swofford, D. L., Maddison, W. P., 1997. Nexus: an extensible file format for systematic information. Systematic Biology 46 (4), 590-621. 12

Mann, C. C., 2009. Addicted to rubber. Science 325 (5940), 564-566. 3

Mears, J. A., 1975. The taxonomy of Parthenium section Partheniastrum DC. (Asteraceae-Ambrosiinae). Phytologia 31, 463-482. 4, 30

Morgulis, A., Gertz, E. M., Schäffer, A. A., Agarwala, R., 2006. A fast and symmetric DUST implementation to mask low-complexity DNA sequences. Journal of Computational Biology 13 (5), 1028-1040. 8

Powers, L., 1942. Seed collection of guayule from Mexico and the Trans-Pecos area (Big Bend Country) of Texas. United States Department of Agriculture, unpublished report. 5

Powers, L., Rollins, R. C., 1945. Reproduction and pollination studies on guayule, Parthenium argentatum Gray and P. incanum H. B. K. Agronomy Journal 37 (2), 96-112. 4

Ray, D. T., Coffelt, T. A., Dierig, D. A., 2005. Breeding guayule for commercial production. Industrial Crops and Products 22 (1), 15-25. 3

Ray, D. T., Dierig, D. A., Thompson, A. E., Coffelt, T. A., 1999. Registration of six guayule germplasms with high yielding ability. Crop Science 39 (1), 300. 16, 17 
Ray, D. T., Foster, M. A., Coffelt, T. A., McMahan, C., 2010. Guayule: culture, breeding and rubber production. In: Singh, B. P. (Ed.), Industrial Crops and Uses. CABI, pp. 384-410. 3

Rivano, F., 1997. South american leaf blight of Hevea I. Variability of Microcyclus ulei pathogenicity. Plantations, Recherche, Développement 4 (2), 104-114. 3

Rollins, R. C., 1946. Interspecific hybridization in Parthenium II. Crosses involving $P$. argentatum, $P$. incanum, $P$. stramonium, $P$. tomentosum and $P$. hysterophorus. American Journal of Botany 33 (1), 21-30. 4

Rollins, R. C., 1950. The guayule rubber plant and its relatives. Contributions from the Gray Herbarium of Harvard University 172, 1-72. 4, 5, 12, 13, 14, $17,20,21,33$

Sanchez, P. L., Costich, D. E., Friebe, B., Coffelt, T. A., Jenks, M. A., Gore, M. A., 2014. Genome size variation in guayule and mariola: fundamental descriptors for polyploid plant taxa. Industrial Crops and Products 54, 1-5. $4,5,6,13,15,16,30,34,36,37$

Shimizu, K., Tsuda, K., 2011. SlideSort: all pairs similarity search for short reads. Bioinformatics 27 (4), 464-470. 10

Spindel, J., Wright, M., Chen, C., Cobb, J., Gage, J., Harrington, S., Lorieux, M., Ahmadi, N., McCouch, S., 2013. Bridging the genotyping gap: using genotyping by sequencing (GBS) to add high-density SNP markers and new value to traditional bi-parental mapping and breeding populations. Theoretical and Applied Genetics 126 (11), 2699-2716. 11

Thompson, A. E., Ray, D. T., 1989. Breeding guayule. In: Janick, J. (Ed.), Plant Breeding Reviews. John Wiley \& Sons, Inc., pp. 93-165. 3, 4, 14, 15, 18 
Tysdal, H. M., Estilai, A., Siddiqui, I. A., Knowles, P. F., 1983. Registration of four guayule germplasms (Reg. No. GP1 to GP4). Crop Science 23 (1), 189. 19

Waln, K., Toukdarian, K., Elder, N., Hanson, G., 1983. Interspecific hybridization. Proceedings of the 3rd International Guayule Conference, 317-326. 19

Wheeler, D. L., Barrett, T., Benson, D. A., Bryant, S. H., Canese, K., Chetvernin, V., Church, D. M., Dicuccio, M., Edgar, R., Federhen, S., Feolo, M., Geer, L. Y., Helmberg, W., Kapustin, Y., Khovayko, O., Landsman, D., Lipman, D. J., Madden, T. L., Maglott, D. R., Miller, V., Ostell, J., Pruitt, K. D., Schuler, G. D., Shumway, M., Sequeira, E., Sherry, S. T., Sirotkin, K., Souvorov, A., Starchenko, G., Tatusov, R. L., Tatusova, T. A., Wagner, L., Yaschenko, E., 2008. Database resources of the National Center for Biotechnology Information. Nucleic Acids Research 36 (Database issue), D13-21. 23 
${ }_{750}$ Tables 


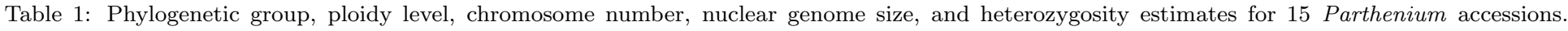

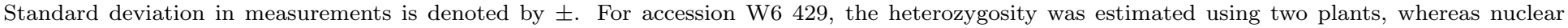

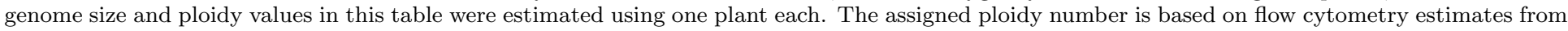

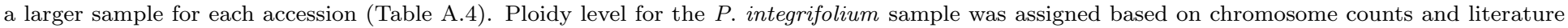
(Mears, 1975).

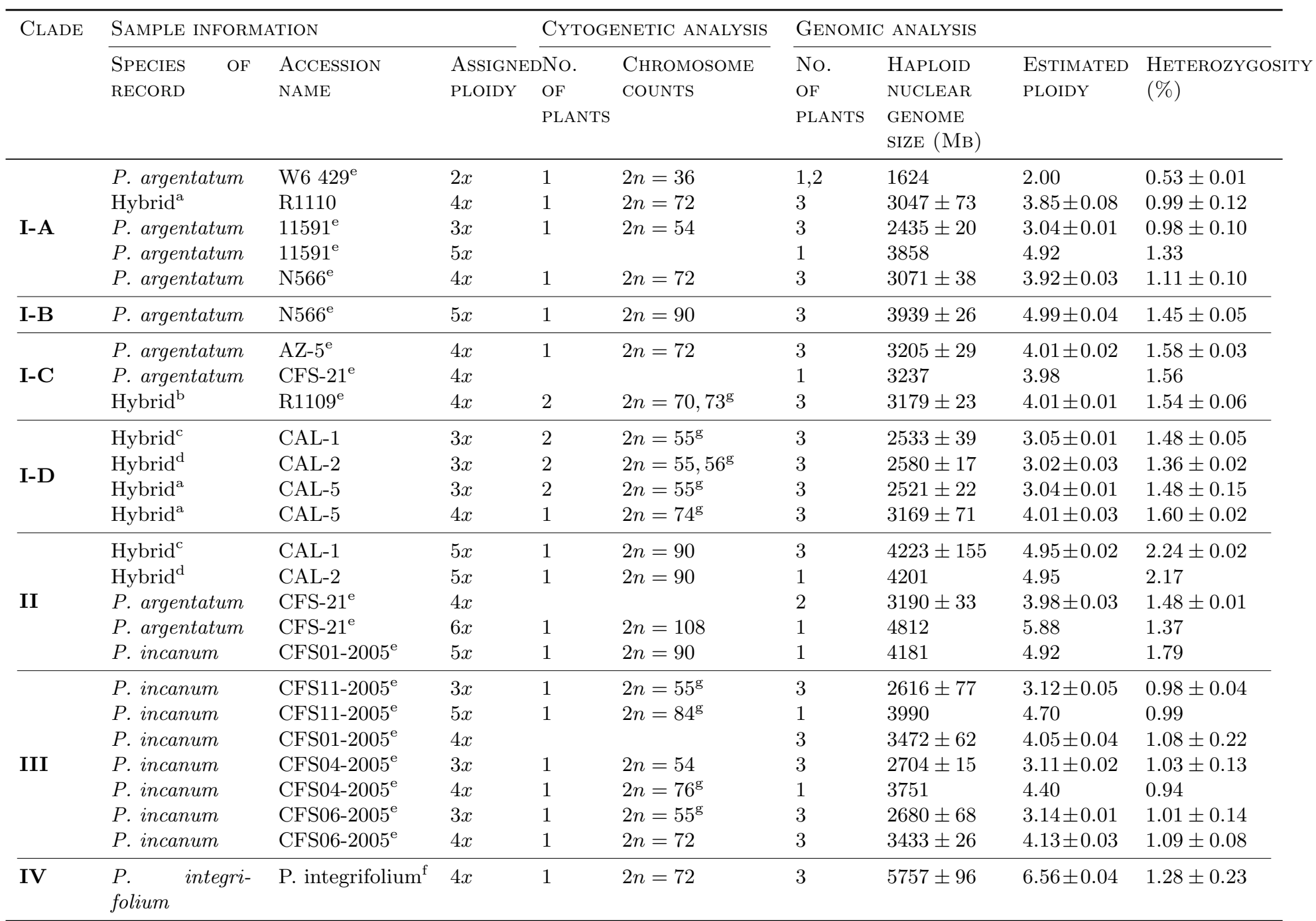

\footnotetext{
${ }^{\text {a }}$ Interspecific hybrid of $P$. argentatum A. Gray and P. tomentosum var. stramonium (Green) Rollins

${ }^{\mathrm{b}}$ Interspecific hybrid of $P$. argentatum A. Gray and $P$. incanum Kunth

${ }^{\mathrm{c}}$ Interspecific hybrid of $P$. argentatum A. Gray and P. tomentosum var. tomentosum DC

${ }^{\mathrm{d}}$ Interspecific hybrid of $P$. argentatum A. Gray and $P$. fruticosum Less.

e Nuclear genome size and ploidy from Sanchez et al. (2014)

${ }^{\mathrm{f}}$ Unofficial accession designation used in this study

g Aneuploid plant(s)
} 


\section{$751 \quad$ Figures}

Figure 1: Sources for Parthenium accessions used in this study. Programs responsible for maintaining the germplasm are indicated by the rectangular blue boxes, and labeled at the top of the figure. The blue horizontal ribbon represents the time axis and indicates the year of accession collection, selection, or generation. Clouds indicate unspecified sources of accessions. The asterisk indicates cryptic hybrids within accessions that were identified by the current study.

Figure 2: Phylogenetic network of 62 plant samples from 15 accessions. Each sample is labeled with the accession name and the assigned ploidy level (number in square brackets). Group I indicates samples of guayule and known interspecific Parthenium hybrids. Group II indicates interspecific hybrid samples newly identified in this study. Group III indicates mariola samples. Group IV indicates wild quinine samples. Capital letters indicate the clades of Group I detailed in section 3.2

Figure 3: Geographical location of origin (white circles) and inferred movement of plant material (blue lines) for the guayule (labels with orange border) and mariola (labels with red border) accessions used in this study. The dashed label border indicates plant material of unknown origin. Asterisks indicate historic accessions from which current cultivars are derived as indicated in Figure 1. Cultivars are indicated by angle brackets around the accession name. 
Figure 1

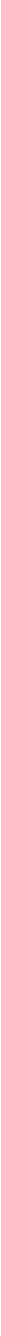

Finer

mergency Rubber Project

sequent Programs 


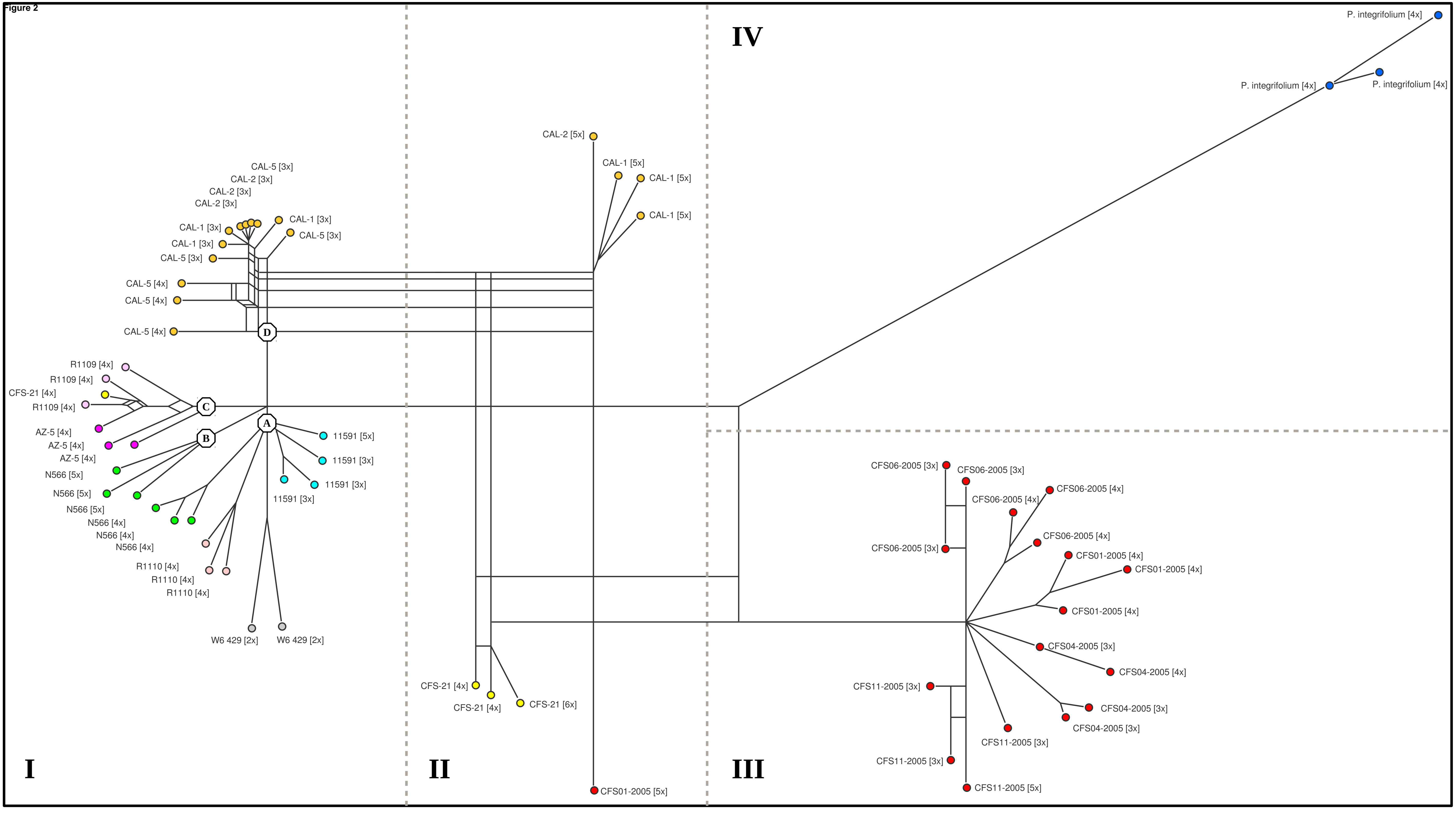


Figure 3

$\langle 11591\rangle,\langle\mathrm{N} 566\rangle$

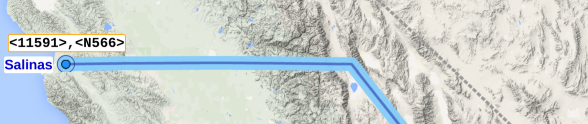

(n)
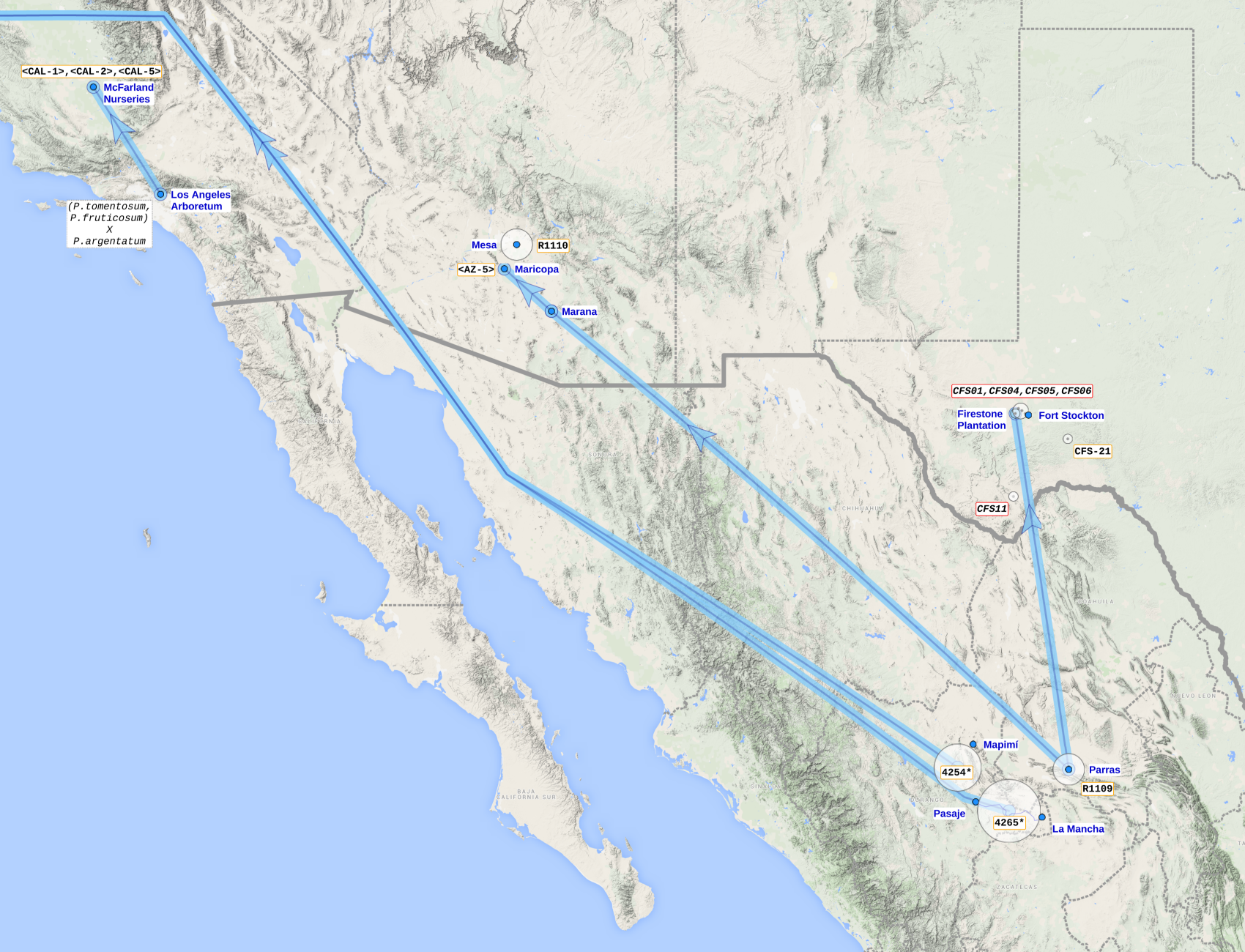